\section{CardioRenal Medicine}

\title{
Subclinical Contrast-Induced Acute Kidney Injury in Patients Undergoing Cerebral Computed Tomography
}

\author{
Andrea Breglia ${ }^{a-c} \quad$ Ilaria Godia, d Grazia Maria Virzi ${ }^{a}$ e

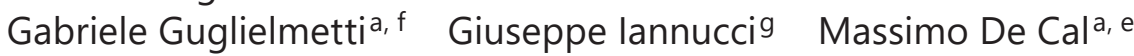 \\ Alessandra Brocca $^{h}$ Mariarosa Carta ${ }^{i}$ Davide Giavarina ${ }^{i}$

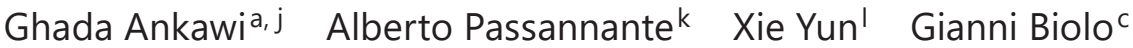 \\ Claudio Ronco ${ }^{a}$, d, e \\ aIRRIV-International Renal Research Institute Vicenza, Vicenza, Italy; ${ }^{b}$ Emergency \\ Department of Arzignano Hospital, Arzignano, Vicenza, Italy; ' ${ }^{\text {Department of Internal }}$ \\ Medicine, University of Trieste, Trieste, Italy; ${ }^{\mathrm{d}}$ Department of Medicine, University of \\ Padova, Padova, Italy; ${ }^{e}$ Department of Nephrology, Dialysis and Transplant, San Bortolo \\ Hospital, Vicenza, Italy; ${ }^{f}$ Nephrology and Kidney Transplantation Unit, Department of \\ Translational Medicine, University of Piemonte Orientale (UPO), "Maggiore della Carità" \\ University Hospital, Novara, Italy; ${ }^{9}$ Department of Neuroradiology, San Bortolo Hospital,

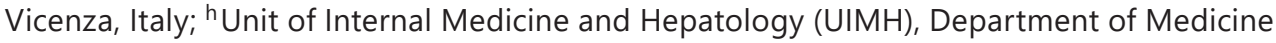 \\ (DIMED), University of Padova, Padova, Italy; 'Department of Laboratory Medicine, \\ San Bortolo Hospital, Vicenza, Italy; 'ंDepartment of Internal Medicine and Nephrology, \\ King Abdulaziz University, Jeddah, Saudi Arabia; ${ }^{\mathrm{k}}$ Department of Anaesthesia and Intensive \\ Care, University of Trieste, Trieste, Italy; 'Department of Nephrology, Xin Hua Hospital \\ affiliated to Shanghai Jiaotong University School of Medicine, Shanghai, China
}

\section{Keywords}

Acute kidney injury · Contrast medium · Biomarkers

\begin{abstract}
Introduction: The nephrotoxicity of modern contrast media remains controversial. Novel biomarkers of kidney damage may help in identifying a subclinical structural renal injury not revealed by widely used markers of kidney function. Objective: The aim of this study was to investigate clinical (contrast-induced acute kidney injury [Cl-AKI]) and subclinical $\mathrm{Cl}-\mathrm{AKI}(\mathrm{SCl}-$ AKI) after intra-arterial administration of lodixanol and lopamidol in patients with an estimated glomerular filtration rate (eGFR) $\geq 60 \mathrm{~mL} / \mathrm{min} / 1.73 \mathrm{~m}^{2}$. Methods: This is a prospective ob-
\end{abstract}

A.B. and I.G. contributed equally to this manuscript. 
servational monocentric study. Urinary sample was collected at 4-8 h after contrast medium exposure to measure neutrophil gelatinase associated lipocalin (NGAL) and the product tissue inhibitor of metalloproteinase-2 and insulin-like growth factor-binding protein 7 ([TIMP-2] $\times$ [IGFBP7]), while blood samples were collected at 24 and $48 \mathrm{~h}$ after exposure to measure serum creatinine. Results: One hundred patients were enrolled, of whom 53 were exposed to lodixanol and 47 to lopamidol. Patients in lodixanol and lopamidol groups were comparable in terms of demographics, pre-procedural and procedural data. No patient developed $\mathrm{Cl}-\mathrm{AKI}$ according KDIGO criteria, while 13 patients reported SCI-AKI after exposure to iodine-based medium contrast (3 patients in lodixanol group and 10 patients in lopamidol group), defined by positive results of NGAL and/or [TIMP-2] $\times$ [IGFBP7]. A positive correlation was found between NGAL and [TIMP-2] $\times$ [IGFBP7] in the analysed population (Spearman's rho 0.49, $p<$ 0.001). In logistic regression analysis, lopamidol exposure showed higher risk for SCl-AKI compared to lodixanol (OR 4.5 [95\% Cl 1.16-17.52], $p=0.030$ ), even after controlling for eGFR and volume of contrast medium used. Conclusions: This study showed that intra-arterial modern contrast media administration may have a nephrotoxic effect in a population without preexisting chronic kidney disease. Further investigations on larger scale are warranted to confirm if lopamidol exposed patients to increased risk of SCI-AKI compared to lodixanol.

(c) 2020 The Author(s)

Published by S. Karger AG, Basel

\section{Introduction}

Contrast-induced acute kidney injury (CI-AKI), previously known as contrast-induced nephropathy, occurs in up to $30 \%$ of patients who received intravascular iodinated contrast media and is generally considered to be the third most common cause of hospital-acquired AKI [1].

It is characterized by a decrease in renal function that occurs within days after the intravascular administration of iodinated contrast medium. Definition of CI-AKI relies on serum creatinine [2,3], a marker notoriously insensitive in detecting renal injury; a rise in serum creatinine may in fact become evident only when $50 \%$ of the renal mass or more is compromised [4].

Several AKI biomarkers have been identified [5] and their value lies in the ability to provide information particularly in patients without traditional signs of impaired renal function. Therefore, we recently proposed a new approach to CI-AKI, that included the use of biomarkers to bring to light subclinical insult after exposure to contrast agents [6].

To support this new approach, both neutrophil gelatinase associated lipocalin (NGAL) and urinary product of tissue inhibitor of metalloproteinase-2 (TIMP-2) and insulin-like growth factor-binding protein 7 (IGFBP7; [TIMP-2] × [IGFBP7]) have been shown to identify a subclinical renal injury at increased risk for adverse outcomes [7, 8]. As such, adoption of preventive or protective strategies may limit development and progression of AKI.

The aim of this study was to investigate CI-AKI and subclinical CI-AKI (SCI-AKI) occurrence after intra-arterial administration of contrast-medium in patients with eGFR $\geq 60 \mathrm{~mL} /$ $\min / 1.73 \mathrm{~m}^{2}$.

We hypothesized that AKI biomarkers may reveal the structural renal damage associated with intra-arterial administration of contrast media even in patients at low risk.

\section{Materials and Methods}

Study Design and Participants

This is a prospective observational monocentric study, aiming to evaluate CI-AKI after exposure to 2 different types of iodinated contrast media. 
All in-hospital and ambulatory adult patients who underwent iodinated contrast medium intra-arterial administration during non-emergent diagnostic and/or interventional neuroradiological procedure from February to August 2018 in San Bortolo Hospital were enrolled. Exclusion criteria were: <18 years old; eGFR $<60 \mathrm{~mL} / \mathrm{min} / 1.73 \mathrm{~m}^{2}$; allergy to iodine and other composition of contrast medium; severe hyperthyroidism; pregnancy.

Contrast Media Types

At our institution, 2 contrast medium types were used indistinctly during the study period: (1) Iodixanol (Visipaque 320, GE Healthcare, Chicago, IL, USA), an iso-osmolar contrast medium (IOCM); (2) Iopamidol (Isovue 370, Bracco Diagnostics Inc, Monroe Township, NJ, USA), a low-osmolar contrast medium (LOCM).

\section{Data Collection and Measurements}

Clinical data, information on medical history, laboratory parameters and type and volume of contrast medium used were evaluated and collected. Mehran risk stratification score was calculated to identify patients at higher risk of developing AKI [9].

Urinary sample for NGAL and [TIMP-2] × [IGFBP7] measurements were obtained in the first $4-8 \mathrm{~h}$ after exposure to contrast agents. Serum samples for measurement of serum creatinine were collected at 24 and $48 \mathrm{~h}$ after the exposure. Serum creatinine was measured with an enzymatic method, Isotope dilution mass spectrometry was traceable by an automatic analyzer (Dimension Vista, Siemens Healthcare, Tarrytown, NY, USA), and estimated glomerular filtration rate (eGFR) was calculated with the CKD-EPI equation [10].

Urine samples were analyzed for biomarkers by technicians blinded to clinical date. [TIMP-2] × [IGFBP7] was analyzed by NephroCheck ${ }^{\circledR}$ (Astute Medical, San Diego, CA, USA). The ASTUTE140 Meter automatically multiplied the concentrations of the 2 biomarkers together and divided this product by 1,000 to report a single numerical test result with units of $(\mathrm{ng} / \mathrm{mL})^{2} / 1,000$.

NGAL was analyzed by Architect (Abbott Laboratories, North Chicago, IL, USA). Architect NGAL assay utilizes a non-competitive, sandwich format with chemiluminescent signal detection. The measuring interval of the ARCHITECT Urine NGAL assay is $10-1,500 \mathrm{ng} / \mathrm{mL}$.

\section{Definitions}

CI-AKI was defined according to KDIGO consensus criteria [3] as the increase in serum creatinine by $\geq 0.3 \mathrm{mg} / \mathrm{dL}(\geq 26.5 \mu \mathrm{mol} / \mathrm{L})$ or an increase of serum creatinine level 1.5 times the baseline. We considered as the baseline value, at least one serum creatinine level within the last 3 months before admission for all patients enrolled in the study. The baseline value was obtained by Biochemical Database of the Central Laboratory of San Bortolo Hospital or by patient's previous clinical check-up.

SCI-AKI was defined as positive results of NGAL and/or [TIMP-2] $\times$ [IGFBP7] values [11]. According to the optimal prediction of AKI, [TIMP-2] $\times$ [IGFBP7] value $>0.3(\mathrm{ng} / \mathrm{mL})^{2} / 1,000$ was defined as positive and a value $\leq 0.3$ was defined as negative [12]. According to the optimal prediction of AKI, NGAL $\geq 90 \mu \mathrm{g} / \mathrm{L}$ was defined as positive and a value $<90 \mu \mathrm{g} / \mathrm{L}$ was defined as negative [13]. NGAL values, which were $<10 \mathrm{ng} / \mathrm{mL}$ and $>1,500 \mathrm{ng} / \mathrm{mL}$, were represented as 10 and 1,500 ng/mL, respectively, for all analyses.

\section{Statistical Analysis}

Primary endpoints were CI-AKI within $48 \mathrm{~h}$ and SCI-AKI at 4-8 h after exposure to contrast-medium. Secondary endpoints were to identify potential risk factors for CI-AKI and SCI-AKI and to evaluate the nephrotoxicity of different types of contrast media, IOCM and LOCM. Continuous variables are presented as median and interquartile range, while categorical variables are described as number and percentage. Continuous data were analysed using Mann-Whitney U test, as appropriate and categorical data were analysed using Pearson Chi-square test. Correlation coefficients were calculated with the Spearman rank correlation coefficient test. Univariate logistic regression analysis was conducted to compare the odds of AKI with different iodinated contrast media. Multivariate analysis was thereafter assessed to adjust the results for potential confounder factors, (including demographics, comorbidities, pre-procedural and procedural parameters) identified as variables with $p$ values $<0.20$ in univariate logistic regression analysis. A $p$ value of $<0.05$ was considered statistically significant.

Statistical analysis was performed using the SPSS Software package. 


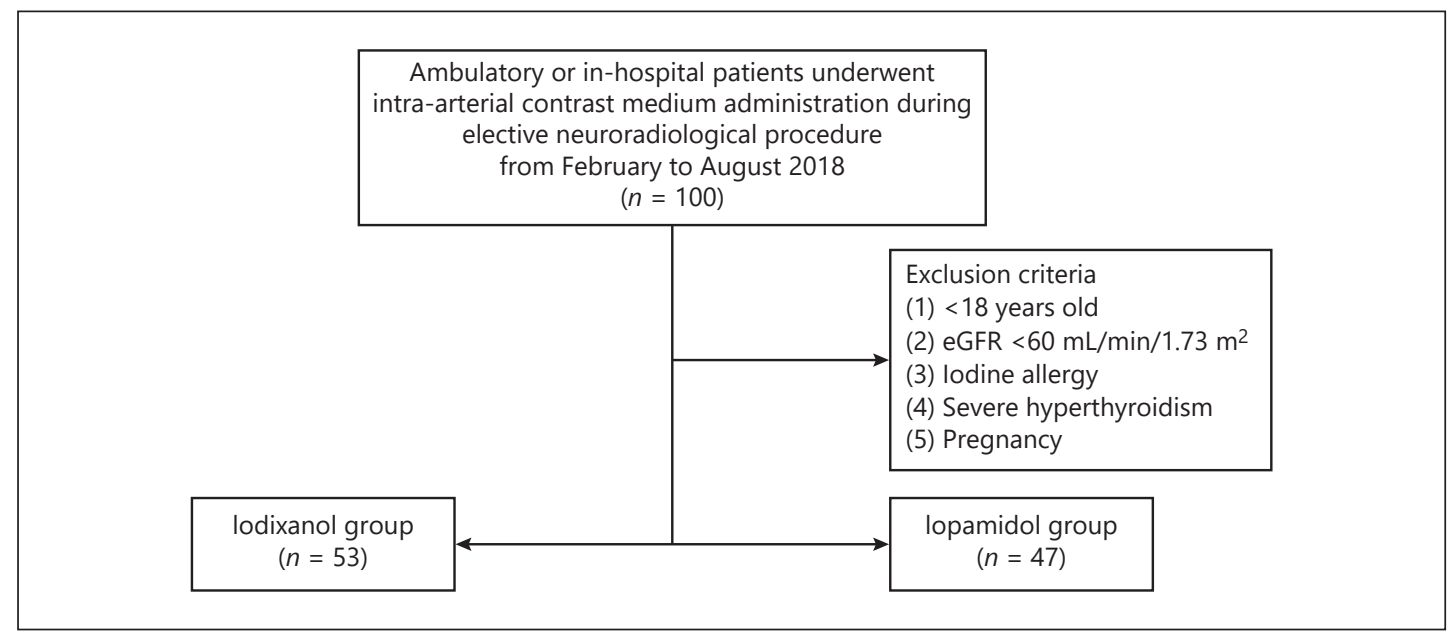

Fig. 1. Flowchart of study design. eGFR, estimated glomerular filtration rate.

Table 1. Demographics, pre-procedural and procedural data in Iodixanol and Iopamidol groups

\begin{tabular}{lcccc}
\hline & All patients & Iodixanol group & Iopamidol group & $p$ values \\
\hline Patients, $n$ & $\mathbf{1 0 0}$ & $\mathbf{5 3}$ & $\mathbf{4 7}$ & \\
Demographics & & & & \\
$\quad$ Age, years & $58(48-72)$ & $57(48-71)$ & $59(49-72)$ & 0.7 \\
$\quad$ BMI & $25(23-27)$ & $25(22-28)$ & $25(23-27)$ & 0.93 \\
$\quad$ Gender, male & $51(51.0)$ & $25(47.2)$ & $26(55.3)$ & 0.42 \\
Pre-procedure parameters & & & & \\
$\quad$ Creatinine, mg/dL & $0.78(0.70-0.90)$ & $0.77(0.65-0.90)$ & $0.78(0.73-0.88)$ & 0.63 \\
$\quad$ eGFR, mL/min/1.73 m ${ }^{2}$ & $91(79-104)$ & $93(81-104)$ & $89(78-103)$ & 0.63 \\
$\quad$ Hemoglobin, g/dL & $14(13-15)$ & $14(13-15)$ & $14(13-15)$ & 0.32 \\
$\quad$ Hematocrit, \% & $42(40-45)$ & $42(39-45)$ & $44(40-45)$ & 0.45 \\
Comorbidities & $1(1.0)$ & $1(1.9)$ & $0(0.0)$ & 0.34 \\
$\quad$ Chronic heart failure, yes & $6(6.0)$ & $5(9.4)$ & $1(2.1)$ & 0.12 \\
$\quad$ Anemia, yes & $4(4.0)$ & $3(5.7)$ & $1(2.1)$ & 0.37 \\
$\quad$ Diabetes, yes & $6(6.0)$ & $4(7.5)$ & $2(4.3)$ & 0.49 \\
$\quad$ Asthma/COPD, yes & $0(0.0)$ & $0(0.0)$ & $0(0.0)$ & 1 \\
$\quad$ Cirrhosis, yes & $1(1-4)$ & $1(1-3)$ & $1(1-4)$ & 0.97 \\
Mehran risk score & $3(3.0)$ & $2(3.8)$ & $1(1.4)$ & 0.55 \\
Nephrotoxic medications & $19(19.0)$ & $10(18.2)$ & $5(10.6)$ & 0.17 \\
$\quad$ Metformin & $11(11.0)$ & $5(9.4)$ & $6(12.8)$ & 0.59 \\
$\quad$ Angiotensin-converting enzyme inhibitors & $1(1.0)$ & $0(0.0)$ & $1(1.4)$ & 0.29 \\
$\quad$ Angiotensin II receptor blocker & $5(5.0)$ & $3(5.7)$ & $2(4.3)$ & 0.75 \\
$\quad$ Non-steroidal anti-inflammatory drugs & $33(33.0)$ & $18(33.9)$ & $15(31.9)$ & 0.83 \\
$\quad$ Loop diuretics & $88(75-114)$ & $85(73-104)$ & $91(80-135)$ & 0.29 \\
Procedure & Interventional & & &
\end{tabular}

Data are reported as number (percentage) for categorical variables and as median (interquartile range) for continuous variables. BMI, body mass index; eGFR, estimated glomerular filtration rate; COPD, chronic obstructive pulmonary disease. 
Fig. 2. Serum creatinine pre- and post-procedure in Iopamidol and Iodixanol groups. The median and interquartile range values of serum creatinine pre-procedure and after 24 and $48 \mathrm{~h}$ after contrast medium exposure did not differ between Iopamidol (orange circle) and Iodixanol (blue triangle) groups.

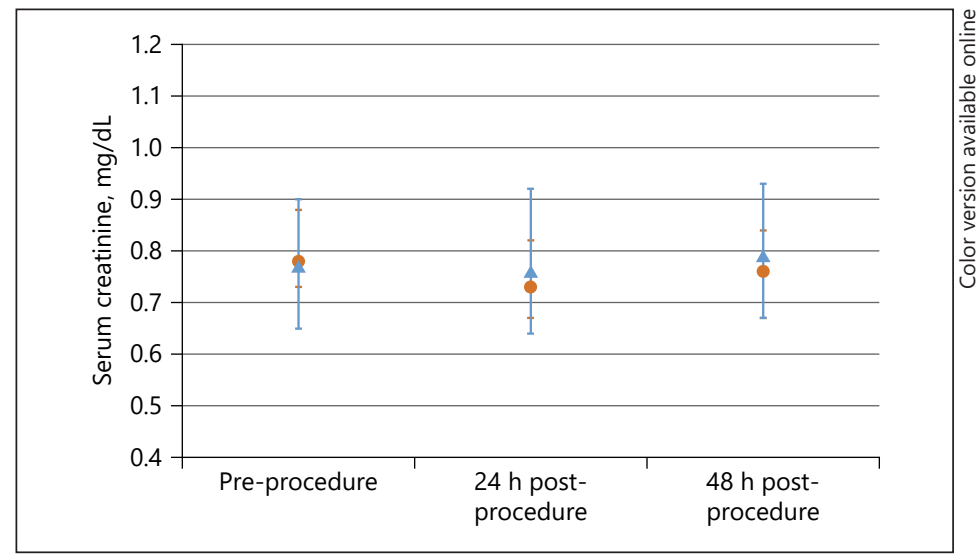

Table 2. Contrast-induced AKI

\begin{tabular}{lcccc}
\hline & All patients & Iodixanol grouplopamidol & $p$ value \\
& & & group & \\
\hline Patients, $n$ & $\mathbf{1 0 0}$ & $\mathbf{5 3}$ & $\mathbf{4 7}$ & \\
CI-AKI & $0(0.0)$ & $0(0.0)$ & $0(0.0)$ & 1.00 \\
SCI-AKI & $13(13.0)$ & $3(5.7)$ & $10(21.3)$ & $0.02^{*}$ \\
NGAL $\geq 90 \mu \mathrm{g} / \mathrm{L}$ & $5(5.0)$ & $1(1.9)$ & $4(8.5)$ & 0.13 \\
[TIMP-2] $\times[\mathrm{IGFBP} 7]>0.3(\mathrm{ng} / \mathrm{mL})^{2} / 1,000$ & $10(10.0)$ & $3(5.7)$ & $7(14.9)$ & 0.12 \\
NGAL $\geq 90 \mu \mathrm{g} / \mathrm{L}$ and $[\mathrm{TIMP}-2] \times[\mathrm{IGFBP7}]$ & & & & \\
$\quad>0.3(\mathrm{ng} / \mathrm{mL})^{2} / 1,000$ & $2(2.0)$ & $1(1.9)$ & $1(2.1)$ & 0.93 \\
\hline
\end{tabular}

Data are reported as number (percentage). * Significant results. CI-AKI, clinical contrast-induced acute kidney injury; SCI-AKI subclinical contrast-induced acute kidney injury; NGAL, neutrophil gelatinase-associated lipocalin; [TIMP-2] $\times$ [IGFBP-7], urinary product of tissue inhibitor mtalloproteinase 2 and insulin growth factor binding protein 7 .

\section{Results}

\section{Subjects Baseline Characteristics}

The study design is shown in Figure 1. We enrolled 100 patients, of whom 53 received Iodixanol and 47 Iopamidol. Characteristics of patients, comorbidities and pre-procedure data were reported in Table 1. No differences were found in terms of age, gender distribution, weight, height, body mass index, pre-procedural serum creatinine, eGFR, haemoglobin and hematocrit. The Iodixanol and Iopamidol groups reported similar percentages of chronic heart failure, anaemia, diabetes mellitus, asthma or chronic obstructive pulmonary disease and cirrhosis. The neuroradiological procedures were conducted with comparable amount of contrast medium in Iodixanol and Iopamidol groups. It is to be noted that a high range of contrast medium was used in each group (min-max 53-326 mL in Iodixanol group and 47-465 mL in Iopamidol group). Mehran risk stratification scores were similar within the 2 groups.

\section{Patient's Outcomes}

The outcomes are detailed in Table 2. No patients developed CI-AKI according to KDIGO consensus criteria. Figure 2 depicts the values of serum creatinine at baseline, 24 and $48 \mathrm{~h}$ after procedure and they were not statistically different between the 2 groups (all $p>0.05$ ). 


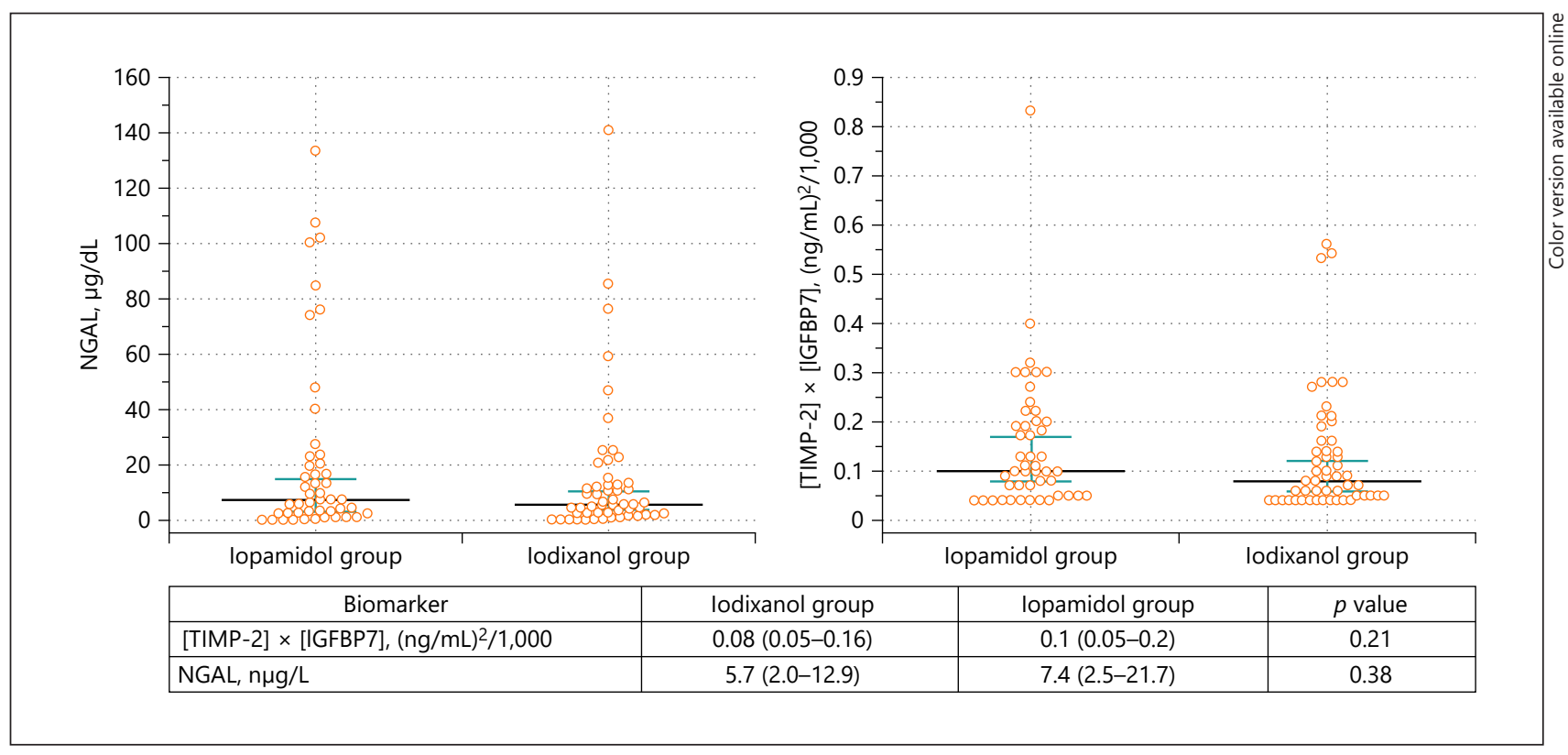

Fig. 3. NGAL and NC values in iopamidol and iodixanol groups. CM1 refers to the Iopamidol group and CM2 refers to the Iodixanol group. NGAL and [TIMP-2] × [IGFBP7] values were similar between the 2 groups. Median and interquartile range values are reported in the table above the charts. NGAL, neutrophil gelatinase associated lipocalin; [TIMP-2] × [IGFBP7], tissue inhibitor of metalloproteinase-2 and insulin-like growth factor-binding protein 7.

Otherwise, SCI-AKI was found in 13 patients ( 3 patients in Iodixanol group and 10 patients in Iopamidol group). In particular, 5 patients showed positive levels of NGAL ( 1 in Iodixanol group, and 4 in Iopamidol group) and 10 patients resulted positive for [TIMP-2] $\times$ [IGFBP7] values ( 3 in Iodixanol group and 7 in Iopamidol group). Only 2 patients were positive for both NGAL and [TIMP-2] × [IGFBP7] values. Biomarkers NGAL and [TIMP-2] × [IGFBP7] resulted similar in the 2 groups (Fig. 3 ).

\section{Subclinical CI-AKI}

We observed that patients exposed to Iopamidol had 3 times higher incidence of SCI-AKI compared to those who exposed to Iodixanol ( 77 vs. $23 \%, p=0.020$ ).

Table 3 compares patients data based on the presence of SCI-AKI. We did not find any difference in terms of age, gender distribution, body mass index, percentages of comorbidities, pre-procedural and procedural data or chronic use of nephrotoxic medications. Mehran risk scores were surprisingly lower in the patients who had SCI-AKI $(p=0.022)$. Patients with positive levels of NGAL reported also higher levels of [TIMP-2] $\times$ [IGFBP7], and patients with [TIMP-2] $\times$ [IGFBP7] positivity had higher NGAL values, as shown in Table 4 .

NGAL and [TIMP-2] $\times$ [IGFBP7]

We assessed Spearman test to study the association between NGAL and [TIMP-2] $\times$ [IGFBP7] in all patients exposed to contrast medium. A positive correlation was found between these 2 biomarkers (Spearman's rho $=0.49, p<0.001$; Fig. 4).

\section{Iopamidol as a Risk Factor for SCI-AKI}

We also performed logistic regression analysis to evaluate the odds of SCI-AKI with Iopamidol relative to Iodixanol exposures. Iopamidol showed a significant higher risk for 
Table 3. Demographics, pre-procedural and procedural data according SCI-AKI

\begin{tabular}{|c|c|c|c|}
\hline & $\begin{array}{l}\text { SCI-AKI } \\
(n=13\end{array}$ & $\begin{array}{l}\text { No SCI-AKI } \\
(n=87)\end{array}$ & $\begin{array}{l}p \\
\text { values }\end{array}$ \\
\hline \multicolumn{4}{|l|}{ Demographics } \\
\hline Age, years & $59(47-69)$ & $58(49-72)$ & 0.65 \\
\hline BMI, $\mathrm{kg} / \mathrm{m}^{2}$ & $25(23-28)$ & $25(23-27)$ & 0.79 \\
\hline Gender, male & $7(53.8)$ & $44(50.6)$ & 0.83 \\
\hline \multicolumn{4}{|l|}{ Parameters pre-procedure } \\
\hline Creatinine, mg/dL & $0.77(0.65-0.81)$ & $0.78(0.70-0.92)$ & 0.17 \\
\hline eGFR, $\mathrm{ml} / \mathrm{min} / 1.73 \mathrm{~m}^{2}$ & 97 (90-104) & $90(78-104)$ & 0.14 \\
\hline Hemoglobin, g/dL & $14(13-15)$ & $14(13-15)$ & 0.99 \\
\hline Hematocrit, $\%$ & $42(38-45)$ & $43(40-45)$ & 0.63 \\
\hline \multicolumn{4}{|l|}{ Comorbidities } \\
\hline Chronic heart failure, yes & $0(0.0)$ & $1(1.0)$ & 0.69 \\
\hline Anemia, yes & $1(7.7)$ & $5(5.7)$ & 0.78 \\
\hline Diabetes, yes & $0(0.0)$ & $4(4.6)$ & 0.43 \\
\hline Asthma/COPD, yes & $1(7.7)$ & $5(5.7)$ & 0.78 \\
\hline Cirrhosis, yes & $0(0.0)$ & $0(0.0)$ & 1.00 \\
\hline Mehran risk score & $1(1-1)$ & $2(1-4)$ & $0.02^{*}$ \\
\hline \multicolumn{4}{|l|}{ Nephrotoxic medications } \\
\hline Metformin & $0(0.0)$ & $3(3.4)$ & 0.50 \\
\hline Angiotensin-converting enzyme inhibitors & $3(23.1)$ & $16(18.4)$ & 0.69 \\
\hline Angiotensin II receptor blocker & $1(7.7)$ & $10(11.5)$ & 0.68 \\
\hline Non-steroidal anti-inflammatory drugs & $0(0.0)$ & $1(1.1)$ & 0.70 \\
\hline Loop diuretics & $1(7.7)$ & $4(4.6)$ & 0.63 \\
\hline \multicolumn{4}{|l|}{ Procedure } \\
\hline Iopamidol administration & $10(76.7)$ & $37(42.5)$ & $0.02 *$ \\
\hline Interventional & $4(30.8)$ & $29(33.3)$ & 0.58 \\
\hline Contrast induced volume, mL & $75(73-90)$ & $91(75-135)$ & 0.06 \\
\hline \multicolumn{4}{|l|}{ Biomarkers } \\
\hline [TIMP-2] $\times$ [IGFBP-7], $(\mathrm{ng} / \mathrm{mL})^{2} / 1,000$ & $0.30(0.28-0.53)$ & $0.08(0.04-0.14)$ & $<0.001^{*}$ \\
\hline NGAL, $\mu \mathrm{g} / \mathrm{L}$ & $76.40(18.10-104.65)$ & $5.20(1.70-12.90)$ & $<0.001^{*}$ \\
\hline
\end{tabular}

Data are reported as number (percentage) for categorical variables and as median (interquartile range) for continuous variables. * Significant results. BMI, body mass index; COPD, chronic obstructive pulmonary disease; eGFR, estimated glomerular filtration rate; SCI-AKI subclinical contrast-induced acute kidney injury; [TIMP-2] × [IGFBP-7], urinary product of tissue inhibitor mtalloproteinase 2 and insulin growth factor binding protein 7; NGAL, neutrophil gelatinase-associated lipocalin.

SCI-AKI compared to iodixanol (OR 4.50 [95\% CI 1.16-17.52], $p=0.030$ ), even after adjusting for volume of contrast medium used and for eGFR values (Table 5).

\section{Discussion}

Contrast-induced nephrotoxicity is a major determinant of community and hospital acquired AKI episodes that lead to hospitalization and prolonged length of stay [14].

It is characterized by a decrease in kidney function that occurs within days after the intravascular administration of iodine-based contrast medium [15]. Pathophysiological mechanisms involve direct nephrotoxic effect of contrast agents to tubular epithelial cells, leading to tubular injury and functional loss, while indirect mechanisms are related to ischemic injury and vasoconstriction [15]. 
Table 4. [TIMP-2] $\times$ [IGFBP7] and NGAL values according NGAL and [TIMP-2] $\times$ [IGFBP7] positivity

\begin{tabular}{llll}
\hline & NGAL positivity & NGAL negativity & $p$ value \\
\hline$\left[\right.$ TIMP-2] $\times\left[\right.$ IGFBP-7], $(\mathrm{ng} / \mathrm{mL})^{2} / 1,000$ & $0.27(0.11-0.43)$ & $0.09(0.05-0.19)$ & $0.02^{*}$ \\
\hline & $\begin{array}{l}\text { [TIMP-2] } \times[\text { IGFBP-7] } \\
\text { positivity }\end{array}$ & $\begin{array}{l}\text { [TIMP-2] } \times[\text { IGFBP-7] } \\
\text { negativity }\end{array}$ & $p$ value \\
\hline NGAL, $\mu \mathrm{g} / \mathrm{L}$ & $57.25(13.92-96.97)$ & $5.55(1.85-13.32)$ & $0.001^{*}$ \\
\hline
\end{tabular}

* Significant results. [TIMP-2] × [IGFBP-7], urinary product of tissue inhibitor mtalloproteinase2 and insulin growth factor binding protein 7; NGAL, neutrophil gelatinase-associated lipocalin.

Fig. 4. Relationship between NGAL and [TIMP-2] $\times$ [IGFBP7]. This scatterplot shows the relationship between the log-transformed NGAL and [TIMP-2] $\times$ [IGFBP7] values. NGAL, neutrophil gelatinase-associated lipocalin.

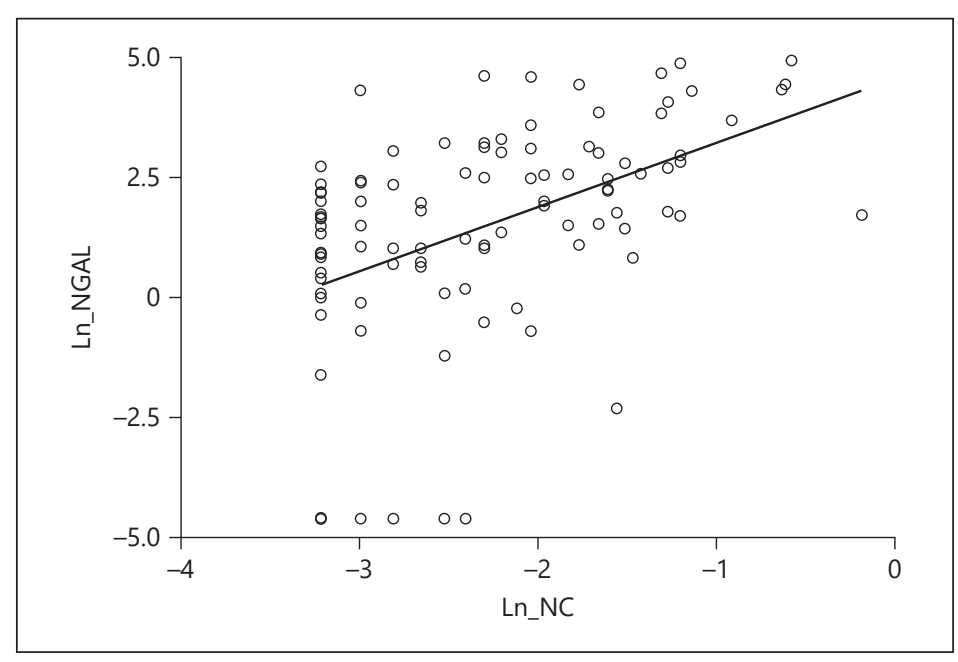

We recently proposed a new concept of CI-AKI, which implies a potentially reversible injury or damage to the kidney occurring within hours or days after the exposure to contrast medium [4]. Even though kidney injury or damage may occur in the absence of kidney dysfunction, the diagnosis of CI-AKI is still made on a change in serum creatinine. However, a rise in serum creatinine or other biomarkers of renal function may become evident only when $50 \%$ or more of the renal mass is compromised [4].

In these circumstances, the adoption of biomarkers of cellular damage and cellular stress, such as NGAL and [TIMP-2] × [IGFBP7], may be useful to identify an early kidney structural damage. Moreover, these biomarkers have been shown to predict both short- and long-term outcomes after AKI [7, 8], suggesting their utility not only in diagnosis but also in AKI prognosis.

Thereafter, we conducted this prospective study to evaluate biomarkers of kidney damage after exposure of 2 commonly used contrast media.

Although not directly examined, we hypothesized that the early use of appropriate biomarkers could potentially help clinicians to identify patients with renal stress and injury who may benefit from strict monitoring or early preventive interventions to limit AKI progression.

The main findings of our study were the subsequent: (1) in our population at low risk of developing CI-AKI, $>10 \%$ of patients had significant elevation of renal injury biomarkers revealing SCI-AKI; (2) patients exposed to Iopamidol had an increased risk of SCI-AKI compared to those who received Iodixanol. 
Table 5. ORs of SCI-AKI

\begin{tabular}{|c|c|c|c|c|c|c|c|c|c|}
\hline & \multicolumn{3}{|c|}{ Univariate analysis } & \multicolumn{6}{|c|}{ Multivariate analysis } \\
\hline & OR & $95 \% \mathrm{CI}$ & $p$ value & OR & $95 \% \mathrm{CI}$ & $p$ value & OR & $95 \% \mathrm{CI}$ & $p$ value \\
\hline Iopamidol & 4.50 & $1.16-17.52$ & $0.03^{*}$ & 4.97 & $1.25-19.80$ & $0.02^{*}$ & 4.99 & $1.24-20.00$ & $0.02^{*}$ \\
\hline Volume of contrast medium & 0.98 & $0.95-1.00$ & 0.10 & 1.60 & $0.91-2.82$ & 0.10 & & & \\
\hline eGFR & 1.03 & $0.99-1.07$ & 0.11 & & & & 1.03 & $1.00-1.08$ & 0.08 \\
\hline
\end{tabular}

* Significant results. SCI-AKI subclinical contrast-induced acute kidney injury; eGFR, estimated glomerular filtration rate.

Recent controlled studies suggested that the risk of CI-AKI was likely non-existent in patients with normal renal function, identified as an eGFR $\geq 60 \mathrm{~mL} / \mathrm{min} / 1.73 \mathrm{~m}^{2}[16,17]$.

In our population, intended to be at low-risk of developing CI-AKI (also confirmed by low Mehran risk stratification scores), we did not observe any case of clinical CI-AKI defined by serum creatinine criteria. Otherwise, SCI-AKI, revealed by elevation of kidney injury biomarkers, was found in $>10 \%$ of patients undergoing intra-arterial contrast medium administration during diagnostic or interventional procedure. To the best of our knowledge this is the first study that suggested a potential nephrotoxicity of modern contrast media in a population without pre-existing chronic kidney disease. As we hypothesized, modern contrast media determined NGAL and [TIMP-2] × [IGFBP7] elevation that may reveal a renal structural insult, while serum creatinine remained silent.

In fact, human kidneys have a significant functional reserve and rise in serum creatinine or reduction in glomerular filtration become evident only when $50 \%$ or more of nephrons is compromised [4].

Additionally, we found a direct correlation between NGAL and [TIMP-2] × [IGFBP7] NGAL is expressed by both proximal tubules and distal nephrons, while TIMP-2 is expressed and secreted by distal tubule cells and IGFBP7 was expressed by proximal cells. Both biomarkers are markers of cellular damage and [TIMP-2] × [IGFBP7] specifically is a marker of cellular stress. A recent experimental study identified a variable expression pattern of TIMP-2 and IGFBP7 within proximal and distal tubules that can be found with and without colocalization of NGAL, suggesting the possibility for differential mechanistic and/or temporal profiles of regulation of these early AKI biomarkers [18]. This may explain how the 2 biomarkers may be complementary in identifying a potential origin of tubular damage and how they may be informative on the pathophysiological mechanisms in the presence of CI-AKI.

This finding supports the hypothesis that both biomarkers increase because of cellular exposure to the toxic effect of iodinated contrast medium, which may have effect both on proximal and distal tubular epithelial cells.

The risk of CI-AKI is influenced by patient- and procedure-related factors [15]. The strongest patient-related risk factor is pre-existing chronic renal disease. Other comorbidities, such as diabetes mellitus, and nephrotoxic comedications further increase the risk of AKI following angiographic procedures $[19,20]$. Several studies have found evidence of a dose-dependent risk increasing with contrast-medium volume administered during the procedure [21, 22].

We analyzed risk factors commonly associated with CI-AKI, such as patient-related (age, comorbidities, chronic use of nephrotoxics) and procedure-related risk factors (volume of contrast medium and interventional procedure), but none of these showed any association with the elevation of AKI biomarkers. Even though in both groups we found cases of SCI-AKI, 
the iopamidol (LOCM) group was exposed 4.5 times more than iodixanol (IOCM) group. This is consistent with another current study in which intra-arterial administration of iodixanol was associated to lower risk of CI-AKI compared to LOCM [23], while in vitro and in vivo studies did not demonstrate significant differences in terms of nephrotoxicity between intravenous administration of low-osmolar and iso-osmolar contrast media [24-27]. Based on our results, other experiments are necessary to better understand if LOCM caused structural damage, not revealed by elevation in serum creatinine.

This study has several limitations. First, this is an observational study conducted in a modest sample size and therefore the results need to be confirmed by prospective randomized trial with a larger sample size. Second, we did not perform baseline measurement of NGAL and [TIMP-2] $\times$ [IGFBP7] prior to contrast medium exposure and this limit the quantification of the injury. Third, we evaluated a timeframe of $48 \mathrm{~h}$ without considering tardive elevation of serum creatinine. This reflects clinical practice where patients admitted for radiological procedure are dismissed up to $48 \mathrm{~h}$ after. Fourth, we did not perform AKI biomarkers in a control group without contrast medium administration.

Nonetheless, this study may be relevant for research in the CI-AKI field and can have clinical implication. This study showed a potential nephrotoxicity of modern contrast media in a population without pre-existing chronic kidney disease and therefore biomarkers should be considered to identify patients with renal damage who may benefit from preventive strategies to limit the progression of the insult. The diagnosis of AKI may include also the subclinical condition and the epidemiology of the syndrome may significantly change. Further investigations on larger scale are warranted to better understand if Iopamidol exposed patients to an increased risk for SCI-AKI compared to Iodixanol.

\section{Acknowledgement}

The authors are grateful to all the fellows of the International Renal Research Institute of Vicenza and the physicians and nurses at Radiology and Nephrology Department of San Bortolo Hospital in Vicenza for the hard work.

\section{Statement of Ethics}

The study was approved by the local Ethics Committee of the San Bortolo Hospital, Vicenza, Italy (Del 83/24 January 2018) and conformed to the Helsinki Declaration. Informed consent was obtained under Italian laws.

\section{Disclosure Statement}

The authors declare no competing interests.

\section{Funding Sources}

Not applicable.

\section{Author Contributions}

A.B., I.G., G.M.V., and C.R. contributed to study design. A.B., G.G., G.I., A.B., G.A., A.P., and X.Y. contributed to data collection. A.B., and G.G. contributed to patient enrolment. M.D.C., A.B., M.C., and D.G. contributed to laboratory work. I.G. and G.M.V. contributed to statistical analysis. A.B., I.G., G.M.V., and C.R. contributed to manuscript writing and editing. All authors reviewed the final manuscript. 


\section{Consent for Publication}

Not applicable.

\section{Availability of Data and Materials}

All relevant data is included in this paper.

\section{References}

1 Fähling M, Seeliger E, Patzak A, Persson PB. Understanding and preventing contrast-induced acute kidney injury. Nat Rev Nephrol. 2017 Mar;13(3):169-80.

2 Stacul F, van der Molen AJ, Reimer P, Webb JA, Thomsen HS, Morcos SK, et al.; Contrast Media Safety Committee of European Society of Urogenital Radiology (ESUR). Contrast induced nephropathy: updated ESUR Contrast Media Safety Committee guidelines. Eur Radiol. 2011 Dec;21(12):2527-41.

3 Khwaja A. KDIGO clinical practice guidelines for acute kidney injury. Nephron Clin Pract. 2012;120(4):c17984.

4 Ronco C, Bellomo R, Kellum J. Understanding renal functional reserve. Intensive Care Med. 2017 Jun;43(6): 917-20.

5 Kashani K, Cheungpasitporn W, Ronco C. Biomarkers of acute kidney injury: the pathway from discovery to clinical adoption. Clin Chem Lab Med. 2017 Jul;55(8):1074-89.

6 Ronco C, Stacul F, McCullough PA. Subclinical acute kidney injury (AKI) due to iodine-based contrast media. Eur Radiol. 2013 Feb;23(2):319-23.

7 Haase M, Devarajan P, Haase-Fielitz A, Bellomo R, Cruz DN, Wagener G, et al. The outcome of neutrophil gelatinase-associated lipocalin-positive subclinical acute kidney injury: a multicenter pooled analysis of prospective studies. J Am Coll Cardiol. 2011 Apr;57(17):1752-61.

8 Koyner JL, Shaw AD, Chawla LS, Hoste EA, Bihorac A, Kashani K, et al.; Sapphire Investigators. Tissue Inhibitor Metalloproteinase-2 (TIMP-2)·IGF-Binding Protein-7 (IGFBP7) Levels Are Associated with Adverse LongTerm Outcomes in Patients with AKI. J Am Soc Nephrol. 2015 Jul;26(7):1747-54.

9 Mehran R, Aymong ED, Nikolsky E, Lasic Z, Iakovou I, Fahy M, et al. A simple risk score for prediction of contrast-induced nephropathy after percutaneous coronary intervention: development and initial validation. J Am Coll Cardiol. 2004 Oct;44(7):1393-9.

10 Levey AS, Inker LA, Coresh J. GFR estimation: from physiology to public health. Am J Kidney Dis. 2014 May; 63(5):820-34.

11 Molitoris BA. Urinary Biomarkers: Alone Are They Enough? J Am Soc Nephrol. 2015 Jul;26(7):1485-8.

12 Hoste EA, McCullough PA, Kashani K, Chawla LS, Joannidis M, Shaw AD, et al.; Sapphire Investigators. Derivation and validation of cutoffs for clinical use of cell cycle arrest biomarkers. Nephrol Dial Transplant. 2014 Nov;29(11):2054-61.

13 Lacquaniti A, Buemi F, Lupica R, Giardina C, Murè G, Arena A, et al. Can neutrophil gelatinase-associated lipocalin help depict early contrast material-induced nephropathy? Radiology. 2013 Apr;267(1):86-93.

14 McCullough PA, Choi JP, Feghali GA, Schussler JM, Stoler RM, Vallabahn RC, et al. Contrast-Induced Acute Kidney Injury. J Am Coll Cardiol. 2016 Sep;68(13):1465-73.

15 Mehran R, Dangas GD, Weisbord SD. Contrast-Associated Acute Kidney Injury. N Engl J Med. 2019 May; 380(22):2146-55.

16 Davenport MS, Khalatbari S, Cohan RH, Dillman JR, Myles JD, Ellis JH. Contrast material-induced nephrotoxicity and intravenous low-osmolality iodinated contrast material: risk stratification by using estimated glomerular filtration rate. Radiology. 2013 Sep;268(3):719-28.

17 McDonald RJ, McDonald JS, Bida JP, Carter RE, Fleming CJ, Misra S, et al. Intravenous contrast material-induced nephropathy: causal or coincident phenomenon? Radiology. 2013 Apr;267(1):106-18.

18 Emlet DR, Pastor-Soler N, Marciszyn A, Wen X, Gomez H, Humphries WH 4th, et al. Insulin-like growth factor binding protein 7 and tissue inhibitor of metalloproteinases-2: differential expression and secretion in human kidney tubule cells. Am J Physiol Renal Physiol. 2017 Feb;312(2):F284-96.

19 Lautin EM, Freeman NJ, Schoenfeld AH, Bakal CW, Haramati N, Friedman AC, et al. Radiocontrast-associated renal dysfunction: incidence and risk factors. AJR Am J Roentgenol. 1991 Jul;157(1):49-58.

20 Cochran ST, Wong WS, Roe DJ. Predicting angiography-induced acute renal function impairment: clinical risk model. AJR Am J Roentgenol. 1983 Nov;141(5):1027-33.

21 Heinrich MC, Häberle L, Müller V, Bautz W, Uder M. Nephrotoxicity of iso-osmolar iodixanol compared with nonionic low-osmolar contrast media: meta-analysis of randomized controlled trials. Radiology. 2009 Jan; 250(1):68-86.

22 McCullough PA, Wolyn R, Rocher LL, Levin RN, O’Neill WW. Acute renal failure after coronary intervention: incidence, risk factors, and relationship to mortality. Am J Med. 1997 Nov;103(5):368-75. 
23 McCullough PA, Brown JR. Effects of Intra-Arterial and Intravenous Iso-Osmolar Contrast Medium (Iodixanol) on the Risk of Contrast-Induced Acute Kidney Injury: A Meta-Analysis. Cardiorenal Med. 2011;1(4):220-34.

24 Heinrich MC, Kuhlmann MK, Grgic A, Heckmann M, Kramann B, Uder M. Cytotoxic effects of ionic high-osmolar, nonionic monomeric, and nonionic iso-osmolar dimeric iodinated contrast media on renal tubular cells in vitro. Radiology. 2005 Jun;235(3):843-9.

25 Bolognese L, Falsini G, Schwenke C, Grotti S, Limbruno U, Liistro F, et al. Impact of iso-osmolar versus lowosmolar contrast agents on contrast-induced nephropathy and tissue reperfusion in unselected patients with ST-segment elevation myocardial infarction undergoing primary percutaneous coronary intervention (from the Contrast Media and Nephrotoxicity Following Primary Angioplasty for Acute Myocardial Infarction [CONTRAST-AMI] Trial). Am J Cardiol. 2012 Jan;109(1):67-74.

26 Solomon RJ, Natarajan MK, Doucet S, Sharma SK, Staniloae CS, Katholi RE, et al.; Investigators of the CARE Study. Cardiac Angiography in Renally Impaired Patients (CARE) study: a randomized double-blind trial of contrast-induced nephropathy in patients with chronic kidney disease. Circulation. 2007 Jun;115(25):318996.

27 Reed M, Meier P, Tamhane UU, Welch KB, Moscucci M, Gurm HS. The relative renal safety of iodixanol compared with low-osmolar contrast media: a meta-analysis of randomized controlled trials. JACC Cardiovasc Interv. 2009 Jul;2(7):645-54. 